\title{
PERFIL EPIDEMIOLÓGICO E NÍVEL DE INDEPENDÊNCIA FUNCIONAL DOS IDOSOS INSTITUCIONALIZADOS NA CASA DE RECUPERAÇÃO DONA ZULMIRA, NO MUNICÍPIO DE GOVERNADOR VALADARES/MG
}

\section{EPIDEMIOLOGICAL PROFILE AND LEVEL OF FUNCTIONAL INDEPENDENCE OF ELDERLY INSTITUTIONALIZED AT THE HALFWAY HOUSE OWNER ZULMIRA, IN THE MUNICIPALITY OF GOVERNADOR VALADARES/MG}

Sabrina Gomes de Morais Mestre, UNIPAC, Brasil.

E-mail: sagomesmorais@ yahoo.com.br

Anaile Duarte Toledo Martins Especialista, UNIVALE, Brasil. E-mail: nanatoledo33@hotmail.com

Francielly Martha Martins Graduada, Brasil.

E-mail: francielly_malta@ hotmail.com

Isabelle Rodrigues Cabral

Graduada, Estados Unidos. E-mail: bellecabral_@hotmail.com

Joana Braz Viana de Oliveira Graduada, Brasil.

E-mail: joanabraz_estetica@ hotmail.com

RESUMO: O objetivo desse estudo foi traçar e avaliar o perfil epidemiológico e o nível de independência funcional em idosos institucionalizados na Casa de Recuperação Dona Zulmira da SSVP. A amostra foi de conveniência com 44 idosos residentes na instituição no período do estudo, que foi realizado em Maio de 2016. O índice de Katz foi utilizado para avaliar os aspectos funcionais relacionados às AVDs. A análise foi descritiva e quantitativa. Predominou 
o gênero feminino; a maior frequência de idade foi entre 60 e 80 anos; identificou-se uma variação do mínimo de um mês e máximo de 25 anos e 6 meses de residência dos idosos sendo o maior percentual residindo de 1 a 5 anos; a Doença de Alzheimer ( $n=8 ; 18,18 \%$ ) e as demais alterações cognitivas $(n=8 ; 18,18 \%)$ apresentaram-se como a de maior prevalência; quanto ao grau de independência funcional dos internos, a maioria dos idosos apresentou um grau elevado de dependência, levando em consideração a necessidade de ajuda não humana, ajuda humana, e a dependência completa em todas as AVDs analisadas, exceto na alimentação que apresentou índice maior de internos independentes. Dentre as AVDs avaliadas, destacou-se a forte correlação entre dependência para transferência e continência. Concluiu-se que se torna basilar ampliar e aprofundar pesquisas com avaliações voltadas para a população idosa em todos os seus aspectos, físicos, mentais, sociais e ambientais, sempre no intuito de contribuir para a melhoria da capacidade funcional.

Palavras-chave: Idosos. Instituição de Longa Permanência; Independência Funcional; Fisioterapia; Atividades de Vida Diária.

ABSTRACT: The objective of this study was to outline and evaluate the epidemiological profile and the level of functional independence in institutionalized elderly at halfway house owner Zulmira of SSVP. The sample of convenience with 44 elderly residents at the institution during the study, which was conducted in May 2016. The Katz index was used to evaluate the functional aspects related to ADLs. The analysis was descriptive and quantitative. Predominated the feminine gender; the highest frequency of age was between 60 and 80 years; We identified a minimum variation of one month and a maximum of 25 years and 6 months of residence of the elderly being the highest percentage living 1-5 years; Alzheimer's disease $(n=8 ; 18.18 \%)$ and other cognitive disorders $(n=8 ; 18.18 \%)$ presented themselves as the most prevalent; the degree of functional independence of the inmates, most of the elderly showed a high degree of dependence, taking into account the need not human aid, human aid, and the complete dependence on all analyzed ADLs except the alimentation with the highest index independent internal. Among the assessed ADLs, highlighted the strong correlation between to transference and continence. It concludes that it is fundamental to broaden and deepen research on focused reviews for the elderly in all its aspects, physical, mental, social and environmental, always in order to contribute to the improvement of functional capacity.

Keywords: Elderly; Long-term care Institution; Functional independence; Physiotherapy; Activities of Daily Living.

\section{INTRODUÇÃO}

De acordo com o Instituto Brasileiro de Geografia e Estatística (IBGE), o número de 
idosos no país saltou de 3 milhões (1960) para 17 milhões (2010). A Pesquisa Nacional por Amostras de Domicílios (PNAD) demonstrou que nesse ritmo a população de idosos alcançará 32 milhões em 2020 (IBGE, 2016).

O município de Governador Valadares em Minas Gerais, localizado na região sudeste, e na mesorregião do Vale do Rio Doce, possui uma população de 263.689 habitantes. Conforme o censo de 2010, o número de idosos entre homens e mulheres com 60 anos ou mais é de 9.275 , correspondendo a aproximadamente 3,5\% da população total do município. Esse contexto demográfico demonstrou não apenas um aumento da longevidade, como também acréscimo na expectativa vida da população brasileira (IBGE, 2010).

A expectativa de vida está entrelaçada com o declínio do estado de saúde físico e mental do idoso. Diante do envelhecimento, o indivíduo fica mais susceptível à incapacidade funcional para realizar as atividades básicas da vida (ABVD) e as Atividades Instrumentais de Vida Diária (AIVD), demonstrando uma interferência direta nas habilidades funcionais do

indivíduo, e em muitos casos gerando incapacidade (LIMA-COSTA, LOYOLA FILHO, MATOS, 2007).

A perda funcional para realização das tarefas diárias, bem o declínio do estado mental (ARAÚJO; CEOLIM, 2007), somada à mudança da dinâmica familiar (CARVALHO; DIAS, 2011), dificuldade de encontrar alguém responsável no cuidado as suas necessidades, aumenta o risco do idoso ser direcionado a uma Instituição de Longa Permanência (CORRÊA et al., 2012).

Sob essa ótica é que Perlini, Leite, Furini (2007) descreveram ser crescente a procura por instituições de longa permanência para idosos (ILPI), uma vez que têm a função de cuidar, suprindo a falta de suporte familiar e social.

A Casa de Recuperação Dona Zulmira da Sociedade de São Vicente de Paulo, onde o estudo foi realizado, é regulada por Leis Federal, Estadual e Municipal, se tornando efetivamente uma ILPI, a partir de setembro de 2005, onde passou a acolher somente idosos 
com 60 (sessenta) anos ou mais, desde que não sejam portadores de doenças contagiosas e /ou dependentes químicos. $\quad$ Essa instituição possui um convênio com a Universidade Vale do Rio Doce (UNIVALE), funcionando como campo de estágio para o curso de Fisioterapia.

Não se tem conhecimento do perfil (epidemiológico, clínico e de independência funcional) da população idosa residente nesta instituição. Assim, questionou-se: a Casa de Recuperação Dona Zulmira apresenta elevado número de idosos institucionalizados dependentes?

Acredita-se que o conhecimento dessa população possa fornecer subsídios que auxiliem na formulação e implementação de Políticas Públicas de atendimento às reais necessidades dos idosos institucionalizados; auxiliar na alocação de recursos que possibilitem uma assistência mais adequada; e ainda contribuir para a melhoria da assistência e da qualidade de vida dos idosos institucionalizados.

Em vista disso, o objetivo deste estudo foi traçar e avaliar o perfil epidemiológico e o nível de independência funcional em idosos institucionalizados na Casa de Recuperação Dona Zulmira da SSVP.

\section{MATERIAIS E MÉTODOS}

\section{Questões éticas}

Trata-se de um estudo do tipo transversal. O presidente da Casa de Recuperação Dona Zulmira da SSVP, recebeu uma carta autorizando assim a realização do estudo. O estudo foi aprovado pelo Comitê de Ética em Pesquisa da Universidade Vale do Rio Doce (CEP n ${ }^{\circ}$ $1.565 .715 / 2016)$

\section{Amostra}

A amostra foi de conveniência e constituída por 44 idosos residentes na instituição no período da concretização deste estudo, ou seja, em Maio de 2016.

\section{Instrumentos}


As informações do perfil epidemiógico dos idosos foram coletadas dos prontuários disponíveis na instituição. Para facilitar a coleta e análise dos dados foi criado um formulário individual, feito no Word, contendo as informações de idade, gênero, condições clinicas (doenças e comorbidades) e tempo de internação.

Para avaliar os aspectos funcionais relacionados às AVDs, foi utilizado o Índice de Katz. Essa escala já foi validada e adaptada para a população brasileira e tem por objetivo avaliar o grau de dependência dos idosos relacionados às atividades básicas de vida diária (ABVDs), como: tomar banho, vestir, ir ao banheiro, realizar transferências, ter continência e alimentar-se. Os dados para a classificação sendo o Índice de Katz foram obtidos pelas acadêmicas do Curso de Fisioterapia da Univale por meio da análise dos prontuários da Fisioterapia com ajuda dos relatos dos profissionais da instituição.

Para a discussão foram utilizados artigos científicos disponíveis nas bases de dados PubMed, Scielo, Periódicos CAPES. O período de busca foi de Abril a Junho de 2016.

\section{Procedimentos de análise}

Foi realizada uma análise descritiva dos dados onde se utilizou estatísticas descritivas básicas, como: frequência, percentual, média, moda, valor mínimo e máximo, e teste de correlação de Pearson.

\section{RESULTADOS}

Participaram deste estudo 44 idosos, dentre eles, 27 mulheres $(61,36 \%)$ e 17 homens $(38,64 \%)$. A maior frequência de idade foi entre 60 e 80 anos sendo que com idade menor que 60 foram $4(9,1 \%)$, com idade entre 60 a 80 foram $27(61,36 \%)$ e com idade igual e maior que 81 anos foram $13(29,54 \%)$. (Tabela 1$)$

Quanto ao tempo de institucionalização identificou-se uma variação do mínimo de um mês e máximo de 25 anos e 6 meses de residência dos idosos sendo o maior percentual residindo de 1 a 5 anos na instituição. Assim, dentre os 44 idosos, 3 (6,81\%) residiam a menos que 1 ano na instituição, $24(54,55 \%)$ de um a cinco anos, $4(9,09 \%)$ de seis a dez 
anos e $13(29,55 \%)$ mais que dez anos. (Tabela 1$)$

\begin{tabular}{cc}
\hline $\begin{array}{c}\text { Idade e tempo de } \\
\text { internação }\end{array}$ & (n) \% \\
\hline
\end{tabular}

\section{Quanto à idade}
$<60$
(04) $9,1 \%$
Entre 60 e 80
(27) $61,36 \%$
$\geq 81$
(13) $29,54 \%$

\section{Tempo de}

\section{institucionalização}

$<1$ ano

$1-5$ anos

(03)

$6,81 \%$

$54,55 \%$

6 a 10 anos

Mais que 10 anos

$9,09 \%$

$29,55 \%$

(04)

(13)

n= número de idosos; \%= percentagem de idosos 
Tabela 1 - Descrição da idade e tempo de internação dos idosos da Casa de Recuperação Dona Zulmira da SSVP/ Governador Valadares/MG, 2016.

Ao serem analisadas as condições clínicas, a Doença de Alzheimer $(n=8 ; 18,18 \%)$ e as demais alterações cognitivas $(n=8 ; 18,18 \%)$ apresentaram-se como a de maior prevalência nos idosos institucionalizados, seguida do Acidente Vascular Encefálico - AVE ( $n=7$; $15,91 \%)$ e de outras patologias Neurológicas $(n=7 ; 15,91 \%)$. Demais condições clínicas como Asma, Câncer, e Insuficiência Venosa foram identificadas ( $\mathrm{n}=7 ; 15,91 \%)$, bem como patologias osteomusculares $(n=5 ; 11,36 \%)$. Dos 44 idosos apenas $2 ; 4,55 \% \%$, não tiveram as patologias definidas (Gráfico 1).

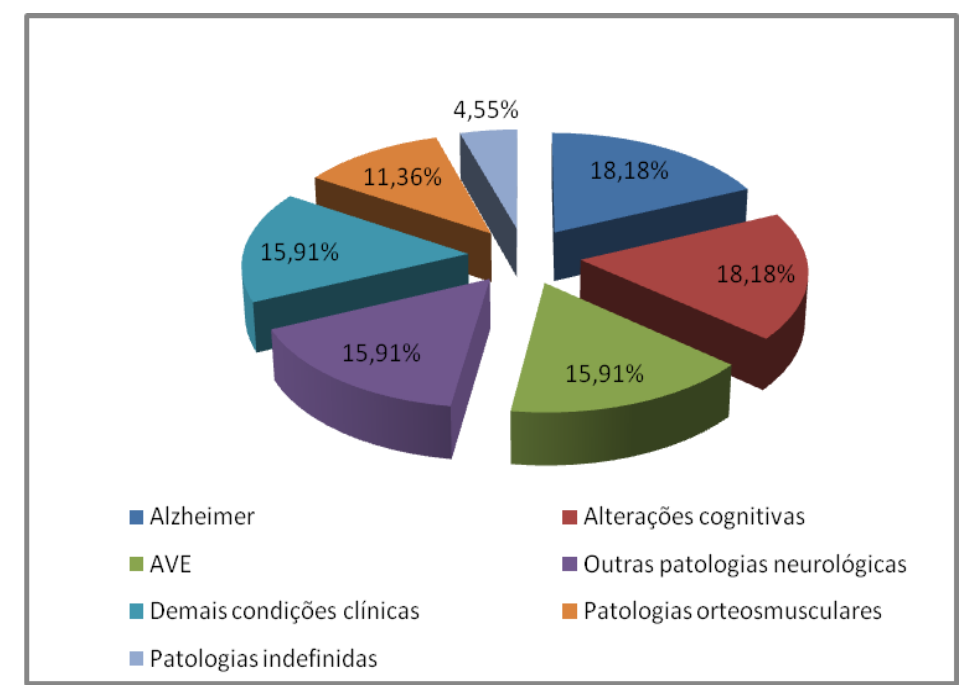


Gráfico 1: Descrição percentual das patologias dos idosos da Casa de Recuperação Dona Zulmira da SSVP/ Governador Valadares/MG, 2016.

Ao serem somados os internos que apresentaram diagnóstico de Doença de Alzheimer com os que apresentaram as outras Alterações Cognitivas foi encontrado um total de $16(36,36 \%)$ internos, e os idosos que apresentaram AVE, somado às outras patologias Neurológicas foram identificados $14(31,82 \%)$. Identificou-se que o maior motivo de internação foram as doenças cognitivas e neurológicas.

Dos 44 idosos participantes, $13(29,55 \%)$ apresentaram comorbidades como Hipertensão Arterial e Diabetes Mellitus.

Com relação ao perfil funcional foram descritos o grau de dependência em cada item avaliado.

Para o banho 15 (34,09\%) necessitavam de ajuda humana e 17 (38,64\%) apresentavam dependência completa nessa atividade; Para vestir $11(25,00 \%)$ necessitavam de ajuda humana e $21(47,73 \%)$ apresentavam dependência completa.

Com relação à Higiene e Continência verificou-se o mesmo grau de dependência nos internos. $18(40,91 \%)$ dos idosos eram considerados independentes, 1 (2,27\%) necessitava de ajuda não humana e $25(56,82 \%)$ apresentavam dependência completa. Já na atividade de transferência observou-se que $20(45,45 \%)$ eram independentes, ou seja, conseguiam subir e descer da cama assim como sentar-se e levantar-se da cadeira sem assistência, porém 4 $(9,09 \%)$ necessitavam de ajuda não humana, 10 (22,73\%) necessitavam da ajuda humana, e $10(22,73 \%)$ tinham dependência completa.

No item Alimentação constatou-se que 33 (75,0\%) idosos conseguiam se alimentar sem assistência. Os demais, $3(6,82 \%)$ recebiam assistência humana para se alimentar e 8 $(18,18 \%)$ apresentavam dependência completa (Tabela 2). 
SAÚdista
SAL VALES 


\section{Banho}

Independente

(12) $27,27 \%$

Necessita de ajuda não humana

(0) $0 \%$

Necessita de ajuda humana

(15) $34,09 \%$

Dependência completa

(17) $38,64 \%$

\section{Vestuário}

Independente

(12) $27,27 \%$

Necessita de ajuda não humana

(0) $0 \%$

Necessita de ajuda humana

(11) $25,00 \%$

Dependência completa

(21) $47,73 \%$

\section{Higiene}

Independente

(18) $40,91 \%$

Necessita de ajuda não humana

(01) $2,27 \%$

Necessita de ajuda humana

(0) $0 \%$

Dependência completa

(25) $56,82 \%$ 


\section{Transferência}

Independente

(20) $45,45 \%$

Necessita de ajuda não humana

(04) $9,09 \%$

Necessita de ajuda humana

(10) $22,73 \%$

Dependência completa

(10) $22,73 \%$

\section{Continência}

Independente

(18) $40,91 \%$

Necessita de ajuda não humana

(01) $2,27 \%$

Necessita de ajuda humana

(0) $0 \%$

Dependência completa

(25) $56,82 \%$

\section{Alimentação}

Independente

(33) $75,00 \%$

Necessita de ajuda não humana

(0) $0 \%$

Necessita de ajuda humana

(03) $6,82 \%$

Dependência completa

(08) $18,18 \%$

$\mathrm{n}=$ número de idosos; \%= percentagem de idosos 
Tabela 2 - Descrição da realização das atividades de vida diária (Índice de Katz). Governador Valadares/MG, 2016.

Conforme descrito, a maioria dos idosos institucionalizados apresentou um grau elevado de dependência, levando em consideração a necessidade de ajuda não humana, ajuda humana, e a dependência completa em todas as atividades de vida diária analisadas, exceto na Alimentação que apresentou índice maior de internos independentes.

Com relação a banho e vestuários 32 (72,73\%) internos apresentaram dependência. Em Higiene e Continência 26 (59,09\%) apresentavam dependência. Para transferências 24 $(54,55 \%)$ apresentavam dependência e para a alimentação apresentando um total de 11 (25\%) dos internos eram dependes (Gráfico 2).

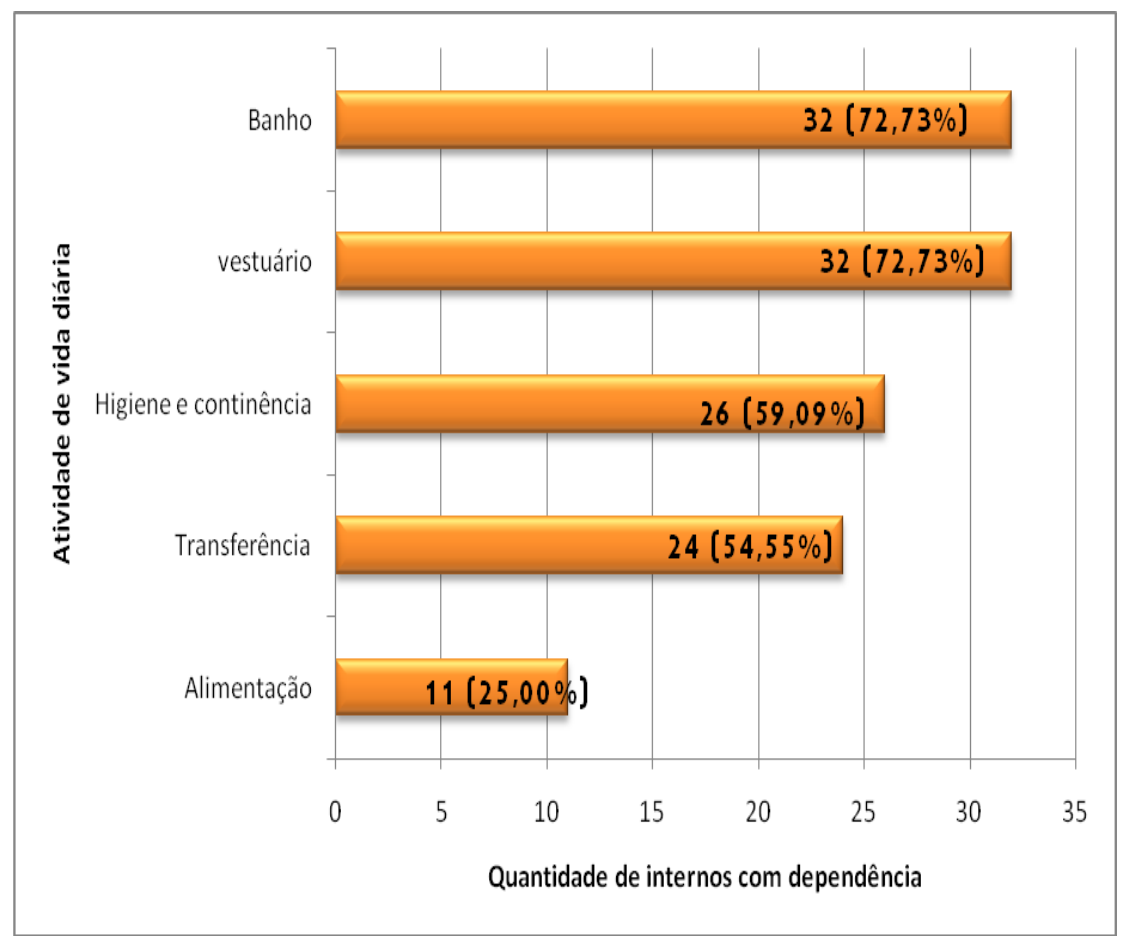


Gráfico 2 - Distribuição de idosos residentes na Casa de Recuperação Dona Zulmira da SSVP de acordo com as atividades da vida diária que apresentam dependência, Governador Valadares/MG, 2016.

Ao observarmos o alto grau de incontinência encontrado nos internos e uma aparente relação com o alto grau de dependência para transferências, foi então realizado análise de correlação visando identificar se realmente havia uma associação entre estas atividades. Através da análise estatística do gráfico 3, sugere-se que há uma forte correlação entre a dependência na transferência e a situação de incontinência apresentada por eles (Gráfico 3).

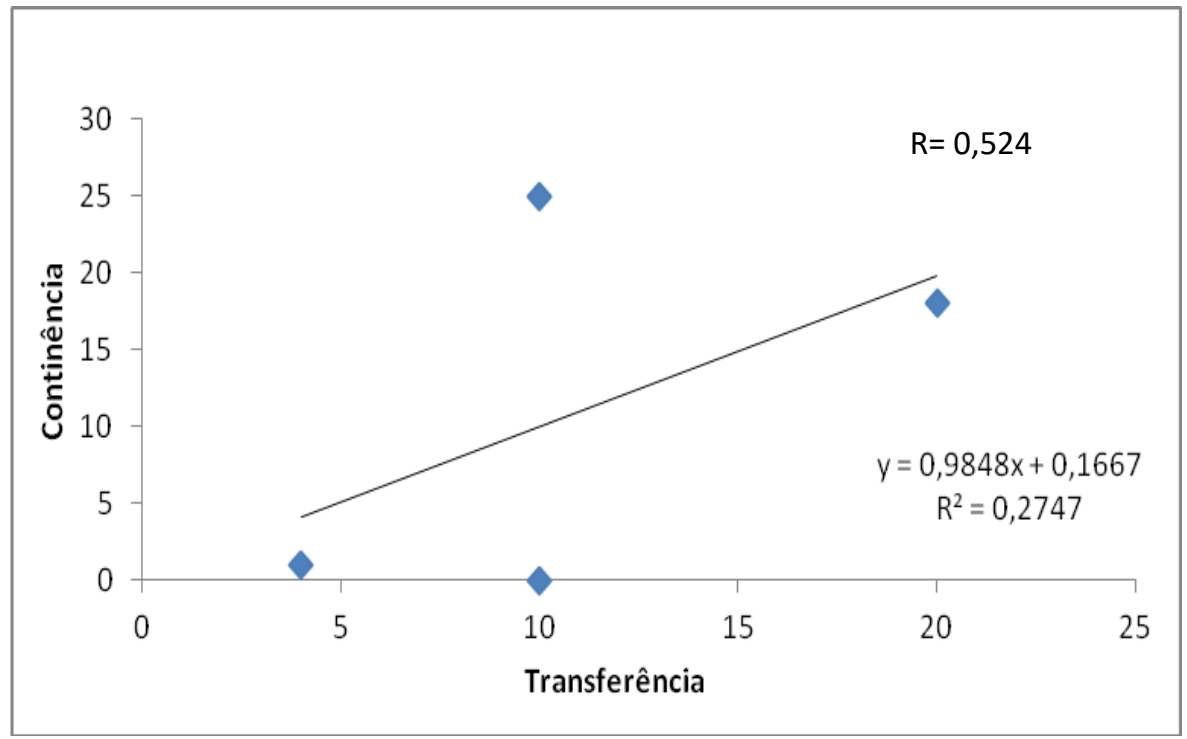

Gráfico 3 - Correlação entre continência e transferência, baseado no resultado obtido através do Índice de Katz.

\section{DISCUSSÃO}

Neste estudo foi encontrada uma predominância do gênero feminino. Estudos apontaram alguns fatores que favorecem a institucionalização das mulheres: pequeno número de filhos ou sem filhos, dificuldades econômicas, têm maior expectativa de vida que os 
homens, sendo que esses apresentam quadro de maior mortalidade o que aumenta o número de mulheres viúvas (ALMEIDA et al. 2015; ALENCAR et al. 2012; LISBOA E CHIANCA, 2012; LIMA E BUENO, 2009). A população de idosos e em especial do gênero feminino, segundo o IBGE (2012) alcançará um alto número até 2050, independente da faixa etária.

A maior quantidade de idosos tinha idade entre 60 e 80 anos e o tempo médio de institucionalização foi grande, sendo a maioria com mais de 5 anos, identificando-se com outros estudos como de Guedes e Silveira (2004), Araújo e Ceolim (2007), Smanioto e Haddad (2011), Alencar et al. (2012), Lisboa e Chianca (2012), Lima e Bueno (2007).

Outros autores apresentaram resultados semelhantes, relatando que os idosos ao residirem em ILPI, por lá permanecem por longos anos ou resto da vida, pois, veem muitas vezes reduzidas às chances de voltar a viver na sua comunidade ou em meio familiar (ALENCAR etal. 2012; LISBOA E CHIANCA 2012). A própria fragilidade do individuo em decorrência do processo de envelhecimento acompanhada de algumas patologias poderá acarretar um aumento no número de pessoas institucionalizadas. Em decorrência da dificuldade em cuidar dos idosos acometidos de alguma patologia, muitas vezes os familiares transferem esse compromisso a uma ILPI. Alencar et al. (2012), Lisboa e Chianca (2012), Podsiadlo e Richardson (1991) descreveram que somados a outros fatores para a institucionalização, estão as doenças crônico degenerativas e suas sequelas, hospitalização recente e dependência para realizar AVDs. Os achados neste estudo demonstraram o predomínio de Alzheimer e outras alterações cognitivas, além de um alto número de idosos dependentes, semelhantes aos descritos na literatura.

$\mathrm{Na}$ avaliação do desempenho das atividades básicas de vida diária constatou-se que poucos idosos eram independentes para realizar algumas das seis tarefas. Observou-se uma dependência maior para o banho e vestuários, seguida da higiene e continência, transferência e alimentação. Esse resultado assemelha-se aos resultados demonstrados por Smanioto e Haddad (2011) e Chaimowicz (1997), que descreveram haver um declínio principalmente nas habilidades mais complexas, com preservação por mais tempo das funções que tem um impacto na sobrevivência. A capacidade de se alimentar sozinho foi a atividade com maior porcentagem de independência neste estudo, não diferente dos achados de Alencar et al. 
(2012), Guedes e Silveira (2004) que também verificaram resultados semelhantes em seus estudos, com maior frequência de independência nesta atividade.

Outros estudos demonstraram resultados contrários à este estudo, pois, evidenciaram um alto grau de independência dos idosos institucionalizados. Entretanto Alencar et al. (2012) teve como critério de exclusão em seu estudo, idosos que apresentassem alterações visuais e/ ou auditivas graves, acamados, instabilidade clinica ou demência grave, e Araújo e Ceolim (2007) adotaram como critério em duas das instituições estudadas, receber apenas idosos capazes de desempenhar sozinho as AVDs, o que poderia ser uma justificativa para parcela significativa de idosos independentes nas AVDs. Regra essa que não se aplica na instituição em que serviu de base para este estudo.

Dentre as AVDs avaliadas, destacou-se a forte correlação entre dependência para transferência e continência. Mancini (2015) descreveu ser frequente a ocorrência de alterações no sistema muscular dos idosos institucionalizados, como fraqueza, desequilíbrio e redução de massa muscular, impossibilitando-os de caminhar, transferir da cama para a cadeira e consequentemente levando-o à incontinência. A continência é descrita como a capacidade normal de uma pessoa de acumular urina e fezes, com controle consciente sobre o tempo e o lugar para urinar e defecar.

Estudos teóricos como o de Huebra et al. (2010) demonstraram que a incontinência urinária pode estar associada à debilidade das extremidades superior e inferior, e à demência, dentre outras situações, o que pôde ser comprovado também neste estudo.

Diante dos resultados apresentados foi possível perceber a necessidade e a devida importância da inserção assistencial de profissionais habilitados da área da saúde, como os Fisioterapeutas. Nesse caso em questão, profissionais Fisioterapeutas poderão trabalhar a prevenção e reabilitação visando manter e/ou recuperar a funcionalidade e consequentemente melhorar a qualidade de vida da população idosa institucionalizada. Destaca-se como essencial a atualização dos prontuários, para que sejam avaliados os aspectos funcionais relacionados às AVDs do idoso, impedindo que a inobservância da instituição seja um agravante para as condições de saúde dessa população. 
Estudos como este tem a pretensão de deixar informações seja qualitativas ou quantitativas. A contribuição é a nível social e acadêmico. Contribuição social, uma vez que contextualiza claramente o assunto de forma que todos possam ler e compreender o processo pelo qual todos os seres humanos alcançarão o envelhecimento e suas dificuldades patológicas e de atividades diárias. Quanto ao nível acadêmico parte-se do princípio de que é um texto que poderá alcançar os profissionais da área de fisioterapia, bem como de outras, e a quem possa interessar, demonstrando as lacunas existentes no trabalho de assistência e trabalho de reabilitação com o idoso.

\section{CONCLUSÃO}

A relação entre o envelhecimento populacional, a implementação de cuidados e a melhor qualidade de vida é uma preocupação que tem motivado a realização de estudos em busca de melhores intervenções de saúde que melhor se aplique às situações das instituições que acolhem os idosos. Assim, percebeu-se que

se torna basilar ampliar e aprofundar pesquisas com avaliações voltadas para a população idosa em todos os seus aspectos, físicos, mentais, sociais e ambientais, sempre no intuito de contribuir para a melhoria da capacidade funcional. Em suma, cumpre assim a finalidade científica deste estudo, que é fornecer subsídios epidemiológicos para provocar reflexão de todos os interessados na melhor qualidade de vida e reversão social da população idosa, principalmente aquela residente na Casa de Recuperação Dona Zulmira da SSVP.

Dessa feita, tornou-se perceptível a necessidade de gerar maior conhecimento do perfil dos idosos institucionalizados, de suas potencialidades funcionais bem como de suas necessidades assistenciais. Sugere-se ampliar estudos nesse sentido, deixando a contribuição reflexiva e urgente de um ambiente acessível no cuidado, prevenção e reabilitação para melhoras na autonomia e independência dos idosos.

\section{REFERÊNCIAS BIBLIOGRÁFICAS}

ALENCAR, M. A et al. Perfil dos idosos residentes em uma instituição de longa permanência. Rev. Bras. Geriatr. Gerontol., Rio de Janeiro. v. 15, n. 4, pp. 785-796, 2012. 
ALMEIDA, R. L. S. instituição de longa permanência para idosos: avaliação das condições de acessibilidade e da funcionalidade dos idosos. Rev. Saúde Com. v.11 n. 2. pp 152-173, 2015.

ARAÚJO, O. P. H.; CEOLIN, M. F.. Avaliação do grau de independência de idosos residentes em instituições de longa permanência. Rev. Esc. Enferm ESP. n. 41, v. 3, , pp 378-85, 2007.

CARVALHO, M. P. R. S.; DIAS, M. O. Adaptação dos Idosos Institucionalizados. Millenium, v. 40, pp. 161-84, 2011.

CASA DE RECUPERAÇÃO DONA ZULMIRA. Disponível em: http://dioc esevaladares.com.br /obrassociais/casa-de-recuperacao-dona-zulmira/ Acesso em: 04 abril 2016.

CHAIMOWICZ F. A saúde dos idosos brasileiros às vésperas do século XXI: problemas, projeções e alternativas. Rev Saúde Pública. v. 31, n. 2, pp. 184-200, 1997.

CORRÊA J.C, et al. Envelhecimento e Percepção Corporal de Idosos Institucionalizados. Rev Br as Geriatr Gerontol, v. 15, n. 1, pp. 63 -8, 2012.

GUEDES, J.M.; SILVEIRA, R. C. Análise da capacidade funcional da população geriátrica institucionalizada na cidade de Passo Fundo-RS. Rev Bras Ciênc Envelhec Hum; v 1, pp. 10-21, 2004.

HUEBRA, R.S et al. Comparação entre Estado Mental, a independência Funcional e Incontinência Urinária em Idosos no interior de Minas Gerais. Revista Inspirar- Movimento e Saúde. v.2, n.6, pp.15-20, nov-dez, 2010.

INSTITUTO BRASILEIRO DE GEOGRAFIA E ESTASTITICA - IBGE. Perfil dos Idosos Responsáveis pelos Domicílios no Brasil: 2010. Disponível em: http://www.censo2010. i b g e . gov.br/ Acesso em: 26 mar. 2016.

INSTITUTO BRASILEITO DE GEOGRAFIA E ESTASTITICA - IBGE. Pesquisa Nacional por Amostra de Domicílios Contínua. 2016. Dispo n í vel em: http://www.ibge.gov.br/home /estatistica /pesqu isa s/pesquisa_resultados.ph p?i d_pesq uias $=1$ 49. Acesso em: 19 de mar. 2016.

LIMA, L. C. V.; BUENO, C. M. L. B. CMLB. Envelhecimento e Gênero: A Vulnerabilidade de Idosas no Brasil. Revista Saúde e Pesquisa. v.2, n. 2, pp. 273-80, mai-ago, 2009.

LIMA-COSTA M.F; LOYOLA FILHO A.I; MATOS D.L. Tendências nas Condições de Saúde e Uso de Serviços de Saúde entre Idosos Brasileiros: um estudo baseado na Pesquisa Nacional por Amostra de Domicílios (1998, 2003). Cad. Saúde Pública. v 23, n10. 2467-78, 2007. 
LISBOA, R. C.; CHIANCA, T. C. M. Perfi 1 epidemiológico, clínico e de independência funcional de uma população idosa institucionalizada. Rev Bras Enferm, Brasilia. v 65, n. 3. pp. 482-7, mai-jun, 2012.

MANCINI, R. B.. Prevalência e fatores associados à baixa capacidade funcional de idosos institucionalizados: uma nova proposta de avaliação composta da capacidade funcional. 93 fls. Dissertação (Especialização em Ciências Médicas) Faculdade de Ciências Médicas da Santa Casa de São Paulo, 2015.

PERLINI, N.M.O.G, LEITE M.T., FURINI A.C. Em Busca de Uma Instituição Para a Pessoa Idosa Morar: Motivos Apontados por Familiares. Rev. Esc. Enferm USP. V 41, n 2. 229236, 2007

PODSIADLO D, RICHARDSON S. The Timed "Up \& Go": a test of Basic Functional Mobility for Frail Elderly Persons. J Am Geriatr Soc. n. 39, pp. 142-148, 1991.

SMANIOTO, F. N.; HADDAD, M. C. F. L. Índice de katz aplicado a idosos institucionalizados. Rev Rene, Fortaleza. v. 12, n. 1, pp. 18-23. jan/mar, 2011. 